\title{
Solvable structures for evolution PDEs admitting differential constraints
}

\author{
Francesco De Vecchi, Paola Morando ${ }^{\dagger}$
}

September 24, 2018

Mathematics Subject Classification: 58J70, 35B06

Keywords: Differential constraints, Partial differential equations, Solvable structures

\begin{abstract}
Solvable structures are exploited in order to find families of explicit solutions to evolution PDEs admitting suitable differential constraints. The effectiveness of the method is verified on several explicit examples.
\end{abstract}

\section{Introduction}

In last decades a great interest has been devoted to symmetry reduction methods for both ordinary and partial differential equations and in recent times several Authors provided different kinds of generalizations of the classical results of Lie and Cartan. This led to the development of new techniques which have given a significant improvement to the subject (see, e.g., 6, 8, 10, 11, 20, 22]).

In particular, the geometric approach based on jet bundles allows the description of an $r$-order ordinary differential equation (ODE) as a finite dimensional submanifold $\mathcal{E}$ of a suitable jet space $J^{r}\left(M, \mathbb{R}^{m}\right)$ ([16, 19, 25]). In this setting, the knowledge of a symmetry for the ODE leads to reduce by one the dimension of the submanifold $\mathcal{E}$ and, under suitable hypotheses, this can be interpreted as a reduction of the order of the ODE. Moreover, using the identification of $J^{r}\left(M, \mathbb{R}^{m}\right)$ with a subspace of the tangent bundle $T\left(J^{r-1}\left(M, \mathbb{R}^{m}\right)\right)$, an $r$-order ODE can be described as a one-dimensional distribution of vector fields on $J^{r-1}\left(M, \mathbb{R}^{m}\right)$. If a solvable $r$-dimensional algebra of symmetries for this distribution is known, the solution to the ODE can be obtained by quadratures. Solvable structures provide an extension of this classical result, significantly enlarging the class of vector fields which can be used to integrate by quadratures a given ODE and, more in general, an integrable distribution of vector fields

\footnotetext{
* Dipartimento di Matematica, Università degli Studi di Milano, via Saldini 50, 20133 Milano (Italy); francesco.devecchi@unimi.it

†DISAA, Università degli Studi di Milano, via Celoria 2, 20133 Milano (Italy); paola.morando@unimi.it
} 
(3, 5, 13, 24]). This approach can be extended to first order scalar partial differential equations (PDEs) as well as to PDEs with one-dimensional Cauchy characteristic space, which are naturally described by a single vector field on a suitable finite-dimensional jet space. In this case, the knowledge of a solvable structure allows the explicit determination of the solutions to the PDE by integrating a given system of closed one-forms (1, 2, 4).

On the other hand, when we consider a system of $m$ evolution PDEs in two independent variables of the form

$$
u_{t}^{i}=f^{i}\left(t, x, u^{j}, u_{x}^{j}, u_{x x}^{j}, \ldots\right),
$$

we have to attach to (11) all its differential consequences and the evolution PDE can be described as an infinite dimensional submanifold $\mathcal{E} \subset J^{\infty}\left(\mathbb{R}^{2}, \mathbb{R}^{m}\right)$ such that $\mathcal{C} \subset T \mathcal{E}$ (here $\mathcal{C}$ denotes the Cartan distribution i.e. the formally integrable distribution on $J^{\infty}\left(\mathbb{R}^{2}, \mathbb{R}^{m}\right)$ generated by the total derivatives). Therefore the knowledge of a symmetry for the PDE does not lead to a reduction of the dimension of the submanifold $\mathcal{E}$ but can be exploited by looking for a special class of solutions which are invariant under the symmetry. This is equivalent to look for the solutions to a new overdetermined system obtained by appending the invariance condition to the original system of PDEs. An interesting generalization of this reduction method is provided by the differential constraints method, consisting in appending to (11) an overdetermined systems of PDEs of the form $\mathcal{L}=\left\{L^{1}\left(x, t, u, u_{\sigma}\right)=0, \ldots, L^{k}\left(x, t, u, u_{\sigma}\right)=0\right\}$ such that the system $\mathcal{L}$ admits a general finite dimensional solution and is compatible with (11). Many reduction methods, such as (conditional) Lie-Bäcklund and non classical symmetry reductions, direct method of Clarkson and Kruskal, Galaktionov's nonlinear separation method and others can be seen as particular instances of differential constraints method (see [7, 9, 12, 14, 15, 17, 18, 20, 23, 26]).

In this paper we use solvable structures in order to obtain families of solutions to systems of evolution PDEs of the form (10) admitting suitable differential constraints. In particular we associate with a differential constraint a finite dimensional submanifold $\mathcal{H} \subset \mathcal{E}$ such that the Cartan distribution $\mathcal{C}$ is tangent to $\mathcal{H}$. Hence solutions to the PDEs (11) satisfying the differential constraints are integral manifolds of $\mathcal{C}_{\mathcal{H}}$ (the Cartan distribution $\mathcal{C}$ restricted to $\mathcal{H}$ ) and the problem of finding particular solutions to the PDE reduces to the problem of finding integral submanifolds of the integrable distribution $\mathcal{C}_{\mathcal{H}}$ on the finite dimensional manifold $\mathcal{H}$. In this setting solvable structures can be successfully exploited to obtain families of explicit solutions to (1).

The paper is organized as follows: is Section 2 we give a geometrical description of differential constraints method for evolution PDEs, in Section 3 we present solvable structures method for evolution PDEs admitting differential constraints and in Section 4 we apply previous results to several explicit examples. 


\section{Differential constraints for evolution PDEs}

Let $M$ be a 2-dimensional manifold and $(x, t)$ be a global coordinate system on $M$. The standard coordinate system for $J^{k}\left(M, \mathbb{R}^{m}\right)$ is $x, t, u^{i}, u_{x}^{i}, u_{t}^{i}, u_{x x}^{i}, \ldots u_{\sigma}^{i}$, where $\sigma$ is a multi-index with $|\sigma| \leq k$ and $u_{\sigma}^{i}$ represents the derivative with respect the variables $x, t$ the number of time given by the multi-index $\sigma=$ $(h, k)$. A coordinate system for $J^{\infty}\left(M, \mathbb{R}^{m}\right)$ is given by $\left(x, t, u^{i}, u_{x}^{i}, \ldots, u_{\sigma}^{i}, \ldots\right)$ without any restriction on the multi-index $\sigma$. It is well known that the Cartan distribution $\mathcal{C}$ on $J^{\infty}\left(M, \mathbb{R}^{m}\right)$ is the 2-dimensional distribution generated by the vector fields

$$
\begin{aligned}
D_{x} & :=\partial_{x}+u_{x}^{i} \partial_{u^{i}}+u_{x x}^{i} \partial_{u_{x}^{i}}+u_{x t}^{i} \partial_{u_{t}^{i}}+\ldots \\
D_{t} & :=\partial_{t}+u_{t}^{i} \partial_{u^{i}}+u_{x t}^{i} \partial_{u_{x}^{i}}+u_{t t}^{i} \partial_{u_{t}^{i}}+\ldots
\end{aligned}
$$

With any system of evolution PDEs of the form

$$
F^{i}:=u_{t}^{i}-f^{i}\left(t, x, u^{j}, u_{x}^{j}, u_{x x}^{j}, \ldots\right)=0,
$$

where $f^{i} \in \mathcal{C}^{\infty}\left(J^{k}\left(M, \mathbb{R}^{m}\right)\right)$ do not depend on the derivatives $u_{t}^{i}, u_{x t}^{i}, \ldots$, it is possible to associate a submanifold $\mathcal{E}$ of $J^{\infty}\left(M, \mathbb{R}^{m}\right)$ such that the restriction $\mathcal{C}_{\mathcal{E}}$ of Cartan distribution to $\mathcal{E}$ satisfies $\mathcal{C}_{\mathcal{E}} \subset T \mathcal{E}$. This means that $\mathcal{E}$ is defined by the equations

$$
F^{i}=0, \quad D_{x}^{l}\left(D_{t}^{r}\left(F^{i}\right)\right)=0 \quad \forall l, r \in \mathbb{N}
$$

and we can consider the natural coordinate system $x, t, u^{i}, u_{r}^{i}$ on $\mathcal{E}$ (here $u_{r}^{i}$ denotes the derivative of the function $u^{i}$ with respect to $x r$ times).

One of the most useful methods for determining particular explicit solutions to a system of evolution PDEs of the form (2) is to reduce it to a system of ODEs. This can be done by enlarging the original system of PDEs appending compatible additional equations (called differential constraints or side conditions, see 14, 15, 17, 20, 21). If we look at the system (2) as a submanifold $\mathcal{E} \subset J^{\infty}\left(M, \mathbb{R}^{m}\right)$ the differential constraints method is equivalent to find a suitable finite-dimensional submanifold $\mathcal{H}$ of $\mathcal{E}$. The particular form of equation (2) and the explicit expression of the generators $\bar{D}_{x}, \bar{D}_{t}$ of $\mathcal{C}_{\mathcal{E}}$

$$
\begin{aligned}
\bar{D}_{x} & =\partial_{x}+u_{x}^{i} \partial_{u^{i}}+u_{x x}^{i} \partial_{u_{x}^{i}}+u_{x x x}^{i} \partial_{u_{x x}^{i}}+\ldots \\
\bar{D}_{t} & =\partial_{t}+f^{i} \partial_{u^{i}}+\bar{D}_{x}\left(f^{i}\right) \partial_{u_{x}^{i}}+\bar{D}_{x}^{2}\left(f^{i}\right) \partial_{u_{x x}^{i}}+\ldots
\end{aligned}
$$

suggest to consider differential constraints $L^{i}\left(x, t, u^{j}, u_{x}^{j}, u_{x x}^{j}, \ldots, u_{n}^{j}\right)=0$ of the form

$$
L^{i}=u_{n_{i}}^{i}-g^{i}\left(t, x, u^{j}, u_{x}^{j}, \ldots\right),
$$

where $\partial_{u_{n}^{i}}\left(g^{j}\right)=0$ for any $n \geq n_{i}$ and to look for a submanifold $\mathcal{H} \subset \mathcal{E}$ defined by

$$
L^{i}=0, \quad \bar{D}_{x}^{r}\left(L^{i}\right)=0 .
$$

In this way we have that $\bar{D}_{x} \in T \mathcal{H}$, but usually $\bar{D}_{t} \notin T \mathcal{H}$.

This corresponds to the fact that, choosing arbitrary functions $g^{i}$, the system 
given by the evolution equations (2) and the differential constraints (4) is not compatible and so the set of solutions is empty. Therefore $\bar{D}_{t} \in T \mathcal{H}$ expresses the compatibility condition between the evolution equations and the differential constraints associated with $\mathcal{H}$.

Definition 1 Let $\mathcal{E}$ be a submanifold of $J^{\infty}\left(M, \mathbb{R}^{m}\right)$ defined by equations (3). A finite-dimensional submanifold $\mathcal{H} \subset \mathcal{E}$ defined by (5) is a constraint submanifold for $\mathcal{E}$ if

$$
\left.\bar{D}_{t}\left(L^{i}\right)\right|_{\mathcal{H}}=0
$$

where the evaluation on $\mathcal{H}$ consists in replacing the expression of $u_{k_{i}}^{i}$, with $k_{i} \geq n_{i}$, in terms of $x, t, u^{i}, u_{h_{i}}^{i}$, with $h_{i}<n_{i}$, using equations (5).

Since $\bar{D}_{t}$ and $\bar{D}_{x}$ commute on $\mathcal{E}$, relations (6) hold if and only if

$$
\left.\bar{D}_{t}\left(\bar{D}_{x}^{r}\left(L^{i}\right)\right)\right|_{\mathcal{H}}=0
$$

and $\mathcal{H} \subset \mathcal{E}$ is a constraint submanifold for $\mathcal{E}$ if and only if $\bar{D}_{t} \in T \mathcal{H}$.

Remark 1 If $\mathcal{H} \subset \mathcal{E}$ is a constraint submanifold for $\mathcal{E}$, the restriction $\mathcal{C}_{\mathcal{H}}$ of the Cartan distribution $\mathcal{C}$ to $\mathcal{H}$ is a completely integrable distribution and any maximal integral submanifold of $\mathcal{C}_{\mathcal{H}}$ corresponds to a common solution to (3) and (5).

Unfortunately equations (6) are usually non-linear PDEs for the functions $g^{i}$ and solving them is as difficult as solving the initial PDE. In this paper we do not address this general problem but we exploit the knowledge of some particular constraint submanifolds and of suitable solvable structures for the restricted Cartan distribution in order to find families of explicit solutions.

\section{Solvable structures and integrability}

In this section we recall some basic definitions and facts about solvable structures in our framework. The reader is referred to [3, 5, 8, 13, 24, for a complete and general discussion of the subject. These results will be used il the next Section to compute families of explicit solutions to evolution PDEs for which a finite-dimensional constraint submanifold is known.

It is well known that, given a $k$-dimensional involutive distribution $K$ on an $n$ dimensional manifold $N$, the knowledge of a solvable $(n-k)$-dimensional algebra $\mathcal{G}$ of nontrivial symmetries for $K$ guarantees that maximal integral submanifolds for $K$ can be found by quadratures. The notion of solvable structure provides a generalization of this classical integrability result, avoiding the use of rectification of vector fields and allowing solutions to be represented in the original coordinates of the problem.

Definition 2 Let $\mathcal{H}$ be an $r$-dimensional constraint submanifold for a system $\mathcal{E}$ of evolution PDEs and $\mathcal{C}_{\mathcal{H}}=\left\langle\tilde{D}_{x}, \tilde{D}_{t}\right\rangle$ be the Cartan distribution restricted 
to $\mathcal{H}$. The vector fields $\left\{X_{1}, X_{2}, \ldots, X_{r-2}\right\}$ are a solvable structure for $\mathcal{C}_{\mathcal{H}}$ if and only if, $\forall h \leq r-2$, the vector field $X_{h}$ is a nontrivial symmetry of $\mathcal{C}_{\mathcal{H}} \oplus\left\langle X_{1}, \ldots, X_{h-1}\right\rangle$.

Theorem 1 Let $\mathcal{H}$ be an $r$-dimensional orientable constraint submanifold of a system $\mathcal{E}$ of evolution PDEs and $\Omega$ be a volume form on $\mathcal{H}$. If $\left\{X_{1}, X_{2}, \ldots, X_{r-2}\right\}$ is a solvable structure for $\mathcal{C}_{\mathcal{H}}$ such that $\mathcal{C}_{\mathcal{H}} \oplus\left\langle X_{1}, \ldots, X_{r-2}\right\rangle=T \mathcal{H}$, then the one-forms

$$
\Omega_{i}:=\frac{\left.\left.\left.\left.\left.\left.\left.X_{1}\right\lrcorner \ldots\right\lrcorner \hat{X}_{i}\right\lrcorner \ldots\right\lrcorner X_{r-2}\right\lrcorner \tilde{D}_{x}\right\lrcorner \tilde{D}_{t}\right\lrcorner \Omega}{\left.\left.\left.\left.\left.X_{1}\right\lrcorner \ldots\right\lrcorner X_{r-2}\right\lrcorner \tilde{D}_{x}\right\lrcorner \tilde{D}_{t}\right\lrcorner \Omega}, \quad i=1, \ldots r-2
$$

satisfy

$$
\begin{aligned}
& d \Omega_{r-2}=0 \\
& d \Omega_{i}=0 \quad \bmod \left(\Omega_{i+1}, \ldots, \Omega_{r-2}\right)
\end{aligned}
$$

and it is possible to explicitly compute $r-2$ first integrals for the 2-dimensional distribution $\mathcal{C}_{\mathcal{H}}$ on $\mathcal{H}$.

The interested reader is referred to the original papers [3, 5, 13, 24] for a proof of this theorem.

Corollary 1 Let $\mathcal{H}$ be an $r$-dimensional orientable constraint submanifold of a system $\mathcal{E}$ of evolution PDEs. If $\left\{X_{1}, X_{2}, \ldots, X_{r-2}\right\}$ is a solvable structure for $\mathcal{C}_{\mathcal{H}}$ such that $\mathcal{C}_{\mathcal{H}} \oplus\left\langle X_{1}, \ldots, X_{r-2}\right\rangle=T \mathcal{H}$, it is possible to explicitly compute a family of solutions to $\mathcal{E}$ depending on $r-2$ parameters.

Proof. The proof is an immediate consequence of Theorem 1 and Remark 1

Remark 2 Theorem 1 can be specialized to the particular case of a distribution $\mathcal{C}_{\mathcal{H}}$ admitting a nontrivial $(r-2)$-dimensional Abelian symmetry algebra $\mathcal{G}=$ $\left\langle X_{1}, \ldots, X_{r-2}\right\rangle$. In this case the function

$$
M=\frac{1}{\left.\left.\left.\left.\left.X_{1}\right\lrcorner X_{2}\right\lrcorner \ldots X_{r-2}\right\lrcorner \tilde{D}_{x}\right\lrcorner \tilde{D}_{t}\right\lrcorner \Omega}
$$

provides an integrating factor for all the one-forms

$$
\left.\left.\left.\left.\left.\left.\left.\beta_{i}:=X_{1}\right\lrcorner \ldots\right\lrcorner \hat{X}_{i}\right\lrcorner \ldots\right\lrcorner X_{r-2}\right\lrcorner \tilde{D}_{x}\right\lrcorner \tilde{D}_{t}\right\lrcorner \Omega
$$

so that each form $\Omega_{i}=M \beta_{i}$ can be separately integrated by quadratures.

\section{Examples}

In this Section we apply previous results to several examples of evolution PDEs in order to show the effectiveness of the combined use of differential constraints and solvable structures to compute families of explicit solutions. 


\subsection{Burgers' equation}

Consider the Burgers' equation

$$
u_{t}=u_{x x}+u_{x}^{2}
$$

and the distribution $\mathcal{C}_{\mathcal{E}}$ generated by

$$
\begin{aligned}
& \bar{D}_{x}=\partial_{x}+u_{x} \partial_{u}+u_{x x} \partial_{u_{x}}+u_{x x x} \partial_{u_{x x}}+\ldots \\
& \bar{D}_{t}=\partial_{t}+\left(u_{x x}+u_{x}^{2}\right) \partial_{u}+\bar{D}_{x}\left(u_{x x}+u_{x}^{2}\right) \partial_{u_{x}}+\bar{D}_{x}^{(2)}\left(u_{x x}+u_{x}^{2}\right) \partial_{u_{x x}}+\ldots
\end{aligned}
$$

If we consider the submanifold $\mathcal{H}$ of $\mathcal{E}$ given by

$$
\mathcal{H}:=\left\{g=u_{x x x}+2 u_{x} u_{x x}=0, \quad \bar{D}_{x}^{(k)} g=0 ; k \in \mathbb{N}\right\},
$$

it is easy to prove that $\mathcal{H}$ is a constraint submanifold for (10), being

$$
\left.\bar{D}_{t}(g)\right|_{\mathcal{H}}=\bar{D}_{x}^{(3)}\left(u_{x x}+u_{x}^{2}\right)+2 u_{x} \bar{D}_{x}^{(2)}\left(u_{x x}+u_{x}^{2}\right)+\left.2 u_{x x} \bar{D}_{x}\left(u_{x x}+u_{x}^{2}\right)\right|_{\mathcal{H}}=0 .
$$

Therefore we can consider the restrictions $\tilde{D}_{x}$ and $\tilde{D}_{t}$ of the vector fields $\bar{D}_{x}$ and $\bar{D}_{t}$ to $\mathcal{H}$. In particular, choosing $\left(x, t, u, u_{x}, u_{x x}\right)$ as coordinates on $\mathcal{H}$, the restricted vector fields are

$$
\begin{aligned}
& \tilde{D}_{x}=\partial_{x}+u_{x} \partial_{u}+u_{x x} \partial_{u_{x}}-2 u_{x} u_{x x} \partial_{u_{x x}} \\
& \tilde{D}_{t}=\partial_{t}+\left(u_{x x}+u_{x}^{2}\right) \partial_{u} .
\end{aligned}
$$

Since $\left\langle\tilde{D}_{x}, \tilde{D}_{t}\right\rangle$ is an involutive distribution on $\mathcal{H}$, Theorem 1 ensures that the non trivial symmetry algebra generated by

$$
X_{1}=\partial_{x}, \quad X_{2}=\partial_{u}, \quad X_{3}=2 t \partial_{t}+x \partial_{x}-u_{x} \partial_{u_{x}}-2 u_{x x} \partial_{u_{x x}}
$$

can be used to compute solutions to (10). In fact, if we take the volume form $\Omega=d x \wedge d t \wedge d u \wedge d u_{x} \wedge d u_{x x}$ on $\mathcal{H}$, the commutation relations

$$
\left[X_{1}, X_{2}\right]=0, \quad\left[X_{1}, X_{3}\right]=X_{1}, \quad\left[X_{2}, X_{3}\right]=0,
$$

ensure that the function

$$
M=\frac{1}{\left.\left.\left.\left.\left.X_{1}\right\lrcorner X_{2}\right\lrcorner X_{3}\right\lrcorner \tilde{D}_{x}\right\lrcorner \tilde{D}_{t}\right\lrcorner \Omega}=\frac{1}{2 u_{x x}\left(u_{x x}+u_{x}^{2}\right)}
$$

provides an integrating factor for the one-forms

$$
\begin{aligned}
& \left.\left.\left.\left.\beta_{2}=X_{1}\right\lrcorner X_{3}\right\lrcorner \tilde{D}_{x}\right\lrcorner \tilde{D}_{t}\right\lrcorner \Omega \\
& \left.\left.\left.\left.\beta_{3}=X_{1}\right\lrcorner X_{2}\right\lrcorner \tilde{D}_{x}\right\lrcorner \tilde{D}_{t}\right\lrcorner \Omega,
\end{aligned}
$$

while the one-form $\left.\left.\left.\left.\Omega_{1}=M \beta_{1}=M X_{2}\right\lrcorner X_{3}\right\lrcorner \tilde{D}_{x}\right\lrcorner \tilde{D}_{t}\right\lrcorner \Omega$ is closed modulo $\Omega_{3}=$ $M \beta_{3}$.

In order to compute explicit solutions we rewrite $\Omega_{3}$ as

$$
\Omega_{3}=\frac{1}{2\left(u_{x x}+u_{x}^{2}\right)} d u_{x x}+\frac{u_{x}}{\left(u_{x x}+u_{x}^{2}\right)} d u_{x}=d\left(\frac{1}{2} \ln \left(u_{x x}+u_{x}^{2}\right)\right)=d F_{3} .
$$


On the level manifolds $F_{3}=\frac{1}{2} \ln \left(u_{x x}+u_{x}^{2}\right)=c_{3}$ we have

$$
u_{x x}=k_{3}^{2}-u_{x}^{2}
$$

with $k_{3}^{2}=e^{2 c_{3}}$. Moreover, using (11), we find

$$
\Omega_{2}=M \beta_{2}=d u-k_{3}^{2} d t-\frac{u_{x}}{k_{3}-u_{x}^{2}} d u_{x}=d\left(u-k_{3}^{2} t+\frac{1}{2} \ln \left(k_{3}^{2}-u_{x}^{2}\right)\right)=d F_{2}
$$

and, on the level manifolds $F_{2}=c_{2}$, we have

$$
u=k_{3}^{2} t-\frac{1}{2} \ln \left(k_{3}^{2}-u_{x}^{2}\right)+c_{2} .
$$

Finally, considering the restriction of the one-form $\Omega_{1}=M \beta_{1}$ to (11), we get

$$
\Omega_{1}=-d x+\frac{1}{k_{3}^{2}-u_{x}^{2}} d u_{x}=d\left(-x+\frac{1}{2 k_{3}} \ln \left(\frac{k_{3}+u_{x}}{k_{3}-u_{x}}\right)\right)=d F_{1}
$$

and, on the level manifolds $F_{1}=c_{1}$, we find

$$
u_{x}=k_{3}\left(\frac{e^{2 k_{3}\left(x+c_{1}\right)}-1}{e^{2 k_{3}\left(x+c_{1}\right)}+1}\right) .
$$

Hence using (12) we obtain the explicit solution to (10) in the form

$$
u=k_{3}^{2} t-\ln \left(\frac{2 k_{3} e^{k_{3}\left(x+c_{1}\right)}}{e^{2 k_{3}\left(x+c_{1}\right)}+1}\right)+c_{2} .
$$

\subsection{Heat equation}

Let consider the heat equation

$$
u_{t}=u_{x x}
$$

and the corresponding distribution generated by

$$
\begin{aligned}
& \bar{D}_{x}=\partial_{x}+u_{x} \partial_{u}+u_{x x} \partial_{u_{x}}+u_{x x x} \partial_{u_{x x}}+\ldots \\
& \bar{D}_{t}=\partial_{t}+u_{x x} \partial_{u}+\bar{D}_{x}\left(u_{x x}\right) \partial_{u_{x}}+\bar{D}_{x}^{(2)}\left(u_{x x}\right) \partial_{u_{x x}}+\ldots
\end{aligned}
$$

The knowledge of a recursion operator for the heat equation provides an infinite family of Lie-Bäcklund symmetries of the form

$$
\begin{aligned}
& X_{1}=u \partial_{u}+u_{x} \partial_{u_{x}}+u_{x x} \partial_{u_{x x}} \ldots, \\
& X_{2}=u_{x} \partial_{u}+u_{x x} \partial_{u_{x}}+u_{x x x} \partial_{u_{x x}} \ldots, \\
& X_{3}=u_{x x} \partial_{u}+u_{x x x} \partial_{u_{x}}+u_{x x x x} \partial_{u_{x x}} \ldots, \\
& \ldots \\
& X_{n}=u_{n-1} \partial_{u}+u_{n} \partial_{u_{x}}+u_{n+1} \partial_{u_{x x}}+\ldots \\
& \ldots
\end{aligned}
$$


In order to reduce to a finite-dimensional manifold, we consider the submanifolds $\mathcal{H}_{n}$ of $\mathcal{E}$ given by

$$
\mathcal{H}_{n}:=\left\{g=u_{n}=0, \quad \bar{D}_{x}^{(k)} g=0 ; \quad k \in \mathbb{N}\right\} .
$$

It is easy to prove that, $\forall n \in \mathbb{N}, \mathcal{H}_{n}$ is a constraint submanifold (corresponding to the Lie-Bäcklund symmetry $X_{n}$ ) for (13) so that we can consider the restrictions $\tilde{D}_{x}$ and $\tilde{D}_{t}$ of the vector fields $\bar{D}_{x}$ and $\bar{D}_{t}$ to $\mathcal{H}_{n}$. In particular, choosing $\left(x, t, u, u_{x}, u_{x x}, \ldots, u_{n-1}\right)$ as coordinates on $\mathcal{H}_{n}$, the restricted vector fields are

$$
\begin{aligned}
& \tilde{D}_{x}=\partial_{x}+u_{x} \partial_{u}+u_{x x} \partial_{u_{x}}+u_{x x x} \partial_{u_{x x}}+\ldots+u_{n-1} \partial_{u_{n-2}} \\
& \tilde{D}_{t}=\partial_{t}+u_{x x} \partial_{u}+u_{x x x} \partial_{u_{x}}+u_{x x x x} \partial_{u_{x x}}+\ldots+u_{n-1} \partial_{u_{n-3}}
\end{aligned}
$$

Since $X_{1}, X_{2}, \ldots X_{n-1}$ are symmetries of $X_{n}$ and generate a non trivial Abelian symmetry algebra for $\left\langle\tilde{D}_{x}, \tilde{D}_{t}\right\rangle$ (when $u_{n-1} \neq 0$ ), Remark 2 ensures that they can be used to compute explicit solutions to (13) for any $n \in \mathbb{N}$.

This example suggests a wide range of possible applications and developments of the proposed method. Indeed a similar procedure can be used whenever a local recursion operator of order one is known, allowing the construction of an infinite family of commuting Lie-Bäcklund symmetries $X_{i}$ (with $i \in \mathbb{N}$ ). In fact, fixing an order $n$ and considering the submanifold $\mathcal{H}_{n}$ corresponding to the vanishing of the generator of $X_{n}$ and its differential consequences, all the vector fields $X_{h}$ with $h<n$ are tangent to $\mathcal{H}_{n}$ and provide (on a suitable submanifold of $\mathcal{H}_{n}$ where they are independent) an Abelian symmetry algebra of suitable

dimension for the distribution generated by the restricted vector fields $\tilde{D}_{x}$ and $\tilde{D}_{t}$.

\subsection{Modified heat equation}

Consider the equation

$$
u_{t}=a u_{x x}+(b x+c)
$$

and the distribution generated by $\bar{D}_{x}$ and

$$
\bar{D}_{t}=\partial_{t}+\bar{D}_{x}\left(a u_{x x}+(b x+c) u\right) \partial_{u_{x}}+\bar{D}_{x}^{(2)}\left(a u_{x x}+(b x+c) u\right) \partial_{u_{x x}}+\ldots
$$

If we take

$$
g=u_{x x x}-\frac{3 u_{x} u_{x x}}{u}+\frac{2 u_{x}^{3}}{u^{2}},
$$

it is easy to verify that the finite-dimensional submanifold $\mathcal{H}$ of $\mathcal{E}$ defined by

$$
\mathcal{H}=\left\{g=0, \bar{D}_{x}^{(k)}(g)=0 ; k \in \mathbb{N}\right\}
$$


is a constraint submanifold for (14). Using $\left(t, x, u, u_{x}, u_{x x}\right)$ as coordinates on $\mathcal{H}$ and denoting by $\tilde{D}_{x}, \tilde{D}_{t}$ the restriction of $\bar{D}_{x}, \bar{D}_{t}$ to $\mathcal{H}$, we have

$$
\begin{aligned}
\tilde{D}_{x}= & \partial_{x}+u_{x} \partial_{u}+u_{x x} \partial_{u_{x}}+\left(\frac{3 u_{x} u_{x x}}{u}-\frac{2 u_{x}^{2}}{u^{2}}\right) \partial_{u_{x x}} \\
\tilde{D}_{t}= & \partial_{t}+\left(a u_{x x}+(b x+c) u\right) \partial_{u}+ \\
& \frac{3 a u u_{x} u_{x x}-2 a u_{x}^{3}+b u^{3}+b u_{x} u^{2} x+c u^{2} u_{x}}{u^{2}} \partial_{u_{x}}+ \\
& \frac{3 a u^{2} u_{x x}^{2}-2 a u_{x}^{4}+2 b u^{3} u_{x}+b u^{3} u_{x x} x+c u^{3} u_{x x}}{u^{3}} \partial_{u_{x x}} .
\end{aligned}
$$

The three vector fields on $\mathcal{H}$

$$
\begin{aligned}
& X_{1}=u \partial_{u}+u_{x} \partial_{u_{x}}+u_{x x} \partial_{u_{x x}} \\
& X_{2}=\partial_{x} \\
& X_{3}=\partial_{t}
\end{aligned}
$$

form a solvable structure for $\tilde{D}_{x}, \tilde{D}_{t}$, being

$$
\begin{gathered}
{\left[X_{1}, X_{2}\right]=\left[X_{1}, X_{3}\right]=\left[X_{2}, X_{3}\right]=0} \\
{\left[X_{1}, \tilde{D}_{x}\right]=\left[X_{2}, \tilde{D}_{x}\right]=\left[X_{3}, \tilde{D}_{x}\right]=0} \\
{\left[X_{1}, \tilde{D}_{t}\right]=\left[X_{3}, \tilde{D}_{t}\right]=0} \\
{\left[X_{2}, \tilde{D}_{t}\right]=b X_{1} .}
\end{gathered}
$$

Since $X_{1}, X_{2}, X_{3}, \tilde{D}_{x}, \tilde{D}_{t}$ are linearly independent on $\mathcal{H}$, we can consider the volume form $\Omega=d t \wedge d x \wedge d u \wedge d u_{x} \wedge d u_{x x}$ on $\mathcal{H}$ and the function $M$ defined by

$$
\begin{aligned}
\frac{1}{M} & \left.\left.\left.\left.\left.=X_{1}\right\lrcorner X_{2}\right\lrcorner X_{3}\right\lrcorner \tilde{D}_{x}\right\lrcorner \tilde{D}_{t}\right\lrcorner \Omega \\
& =2 a \frac{-u^{3} u_{x x}^{3}+3 u^{2} u_{x}^{2} u_{x x}^{2}-3 u u_{x}^{4} u_{x x}+u_{x}^{6}}{u^{3}} .
\end{aligned}
$$

In order to simplify notations we use the new coordinate

$$
v=\frac{u_{x x}}{u}-\frac{u_{x}^{2}}{u^{2}}
$$

instead of $u_{x x}$ and we obtain

$$
M=-\frac{1}{2 a v^{3} u^{3}}
$$

Since $X_{1}, X_{3}, \tilde{D}_{x}, \tilde{D}_{t}$ commute, $M$ is and integrating factor for $\left.\left.\left.\left.\beta_{2}=X_{1}\right\lrcorner X_{3}\right\lrcorner \tilde{D}_{x}\right\lrcorner \tilde{D}_{t}\right\lrcorner \Omega$ and $\left.\left.\left.\left.\beta_{3}=X_{1}\right\lrcorner X_{2}\right\lrcorner \tilde{D}_{x}\right\lrcorner \tilde{D}_{t}\right\lrcorner \Omega$ and

$$
\begin{aligned}
& \Omega_{2}=M \beta_{2}=d x+\frac{u_{x}}{v u^{2}} d u-\frac{1}{v u} d u_{x}+\frac{2 a v u_{x}+b u}{2 a v^{3} u} d v \\
& \Omega_{3}=M \beta_{3}=d t-\frac{1}{2 a v^{2}} d v
\end{aligned}
$$


are closed differential forms. The integral functions of $\Omega_{2}, \Omega_{3}$ are

$$
\begin{aligned}
& F_{2}=x+\frac{4 a v u_{x}-b u}{4 a v^{2} u} \\
& F_{3}=t-\frac{1}{2 a v}
\end{aligned}
$$

and, on the level manifolds $F_{2}=k_{2}, F_{3}=k_{3}$, we get

$$
\begin{aligned}
v & =\frac{1}{2 a\left(k_{2}-t\right)} \\
u_{x} & =\frac{u\left(-a b k_{2}^{2}+2 a b k_{2} t-a b t^{2}-k_{3}+x\right)}{2 a\left(k_{2}-t\right)} .
\end{aligned}
$$

Moreover, from Theorem 1 we have that

$$
\left.\left.\left.\left.\Omega_{1}=M X_{2}\right\lrcorner X_{3}\right\lrcorner \tilde{D}_{x}\right\lrcorner \tilde{D}_{t}\right\lrcorner \Omega
$$

is closed modulo $d F_{2}, d F_{3}$ and integrating $\Omega_{1}$ we find

$$
\begin{aligned}
F_{1}= & -\frac{1}{2} \log \left(t-k_{2}\right)-\log (u)+\left(3 a^{2} b^{2} k_{2}^{3} t-6 a^{2} b^{2} k_{2}^{2} t^{2}+4 a^{2} b^{2} k_{2} t^{3}\right. \\
& -a^{2} b^{2} t^{4}-6 a b k_{2}^{2} x+6 a b k_{2} k_{3} t+12 a b k_{2} t x-6 a b k_{3} t^{2}- \\
& 6 a b t^{2} x+12 a c k_{2} t-12 a c t^{2}-6 k_{3} x+3 x^{2}+ \\
& \left.3 k_{3}^{2}\right) /\left(12 a\left(k_{2}-t\right)\right) .
\end{aligned}
$$

We can solve equation $F_{1}=k_{1}$ with respect to $u$ in order to write the explicit solution

$$
\begin{aligned}
u= & \frac{1}{\sqrt{\left(t-k_{2}\right)}} \exp \left(\frac{x^{2}}{4 a\left(k_{2}-t\right)}+\frac{\left(-k_{3}-a b t^{2}+2 a b k_{2} t-a b k_{2}^{2}\right) x}{2 a\left(k_{2}-t\right)}+\right. \\
& \frac{-a^{2} b^{2} t^{4}+4 a^{2} b^{2} k_{2} t^{3}-\left(6 a^{2} b^{2} k_{2}^{2}+6 a b k_{3}+12 a c\right) t^{2}}{12 a\left(k_{2}-t\right)}+ \\
& \left.\frac{\left(a^{2} b^{2} k_{2}^{3}+2 a b k_{2} k_{3}+4 a c k_{2}\right) t+k_{3}^{2}}{4 a\left(k_{2}-t\right)}-k_{1}\right) .
\end{aligned}
$$

If, for example, we chose $a=1, b=0, c=0, k_{1}=\ln (\sqrt{4 \pi}), k_{2}=0, k_{3}=y$ we obtain

$$
u=\frac{1}{\sqrt{4 \pi t}} \exp \left(\frac{-(x-y)^{2}}{4 t}\right)
$$

that is the well know heat kernel.

\subsection{System of evolution equations}

Consider the following system of evolution equations 


$$
u_{t}=u_{x x}+\frac{1}{2} v^{2}, \quad v_{t}=2 v_{x x}
$$

and the corresponding restricted Cartan distribution generated by

$$
\begin{aligned}
& \bar{D}_{x}=\partial_{x}+u_{x} \partial_{u}+v_{x} \partial_{v}+u_{x x} \partial_{u_{x}}+v_{x x} \partial_{v_{x}}+\ldots \\
& \bar{D}_{t}=\partial_{t}+\left(u_{x x}+\frac{1}{2} v^{2}\right) \partial_{u}+2 v_{x x} \partial_{v}+\bar{D}_{x}\left(u_{x x}+\frac{1}{2} v^{2}\right) \partial_{u_{x}}+\bar{D}_{x}\left(2 v_{x x}\right) \partial_{v_{x}}+\ldots
\end{aligned}
$$

If we consider the submanifold $\mathcal{H}$ of $\mathcal{E}$ given by

$\mathcal{H}:=\left\{g^{1}=u_{x x x}+3 v v_{x}=0, \quad g^{2}=v_{x x x}=0, \quad \bar{D}_{x}^{(k)} g^{1}=0, \quad \bar{D}_{x}^{(k)} g^{2}=0 ; \quad k \in \mathbb{N}\right\}$

it is easy to prove (by explicit computation) that $\mathcal{H}$ is a constraint submanifold for (16). Hence we can restrict the vector fields $\bar{D}_{x}$ and $\bar{D}_{t}$ to $\mathcal{H}$ and, choosing coordinates $\left(x, t, u, v, u_{x}, v_{x}, u_{x x}, v_{x x}\right)$ on $\mathcal{H}$, we find

$$
\begin{aligned}
& \tilde{D}_{x}=\partial_{x}+u_{x} \partial_{u}+v_{x} \partial_{v}+u_{x x} \partial_{u_{x}}+v_{x x} \partial_{v_{x}}-3 v v_{x} \partial_{u_{x x}} \\
& \tilde{D}_{t}=\partial_{t}+\left(u_{x x}+\frac{1}{2} v^{2}\right) \partial_{u}+2 v_{x x} \partial_{v}-2 v v_{x} \partial_{u_{x}}-\left(2 v_{x}^{2}+2 v v_{x x}\right) \partial_{u_{x x}} .
\end{aligned}
$$

Since $\left\langle\tilde{D}_{x}, \tilde{D}_{t}\right\rangle$ is an involutive distribution on $\mathcal{H}$, we can use the solvable structure

$$
\begin{aligned}
& X_{1}=\partial_{t}, \quad X_{2}=\partial_{x}, \quad X_{3}=\partial_{u}, \\
& X_{4}=\partial_{u_{x}}, \quad X_{5}=\partial_{u_{x x}}, \quad X_{6}=\partial_{v_{x x}},
\end{aligned}
$$

in order to find explicit solutions to the system (16). If we take the volume form on $\mathcal{H}$ as $\Omega=d t \wedge d x \wedge d u \wedge d u_{x} \wedge d u_{x x} \wedge d v \wedge d v_{x} \wedge d v_{x x}$ we find

$$
\left.\left.\left.\left.\left.\left.\left.\left.\frac{1}{M}=X_{1}\right\lrcorner X_{2}\right\lrcorner X_{3}\right\lrcorner X_{4}\right\lrcorner X_{5}\right\lrcorner X_{6}\right\lrcorner \tilde{D}_{x}\right\lrcorner \tilde{D}_{t}\right\lrcorner \Omega=-2 v_{x x}^{2}
$$

and we get the closed one-form

$$
\left.\left.\left.\left.\left.\left.\left.\Omega_{6}=M X_{1}\right\lrcorner X_{2}\right\lrcorner X_{3}\right\lrcorner X_{4}\right\lrcorner X_{5}\right\lrcorner \tilde{D}_{x}\right\lrcorner \tilde{D}_{t}\right\lrcorner \Omega=d v_{x x}=d F_{6} .
$$

Moreover, on the level manifolds $F_{6}=c_{6}$, the one-form

$$
\begin{aligned}
\Omega_{5} & \left.\left.\left.\left.\left.\left.\left.=M X_{1}\right\lrcorner X_{2}\right\lrcorner X_{3}\right\lrcorner X_{4}\right\lrcorner X_{6}\right\lrcorner \tilde{D}_{x}\right\lrcorner \tilde{D}_{t}\right\lrcorner \Omega \\
& =\frac{c_{6}^{2} d u_{x x}+\left(c_{6}^{2} v+c_{6} v_{x}^{2}\right) d v+\left(2 c_{6} v v_{x}-v_{x}^{3}\right) d v_{x}}{c_{6}^{2}}
\end{aligned}
$$

is closed and

$$
\Omega_{5}=d F_{5}=d\left(u_{x x}-\frac{v_{x}^{4}}{4 c_{6}^{2}}+\frac{v^{2}}{2}+\frac{v v_{x}^{2}}{c_{6}}\right) .
$$

Furthermore, if we restrict to $F_{5}=c_{5}, F_{6}=c_{6}$, the one-form

$$
\begin{aligned}
\Omega_{4} & \left.\left.\left.\left.\left.\left.\left.=M X_{1}\right\lrcorner X_{2}\right\lrcorner X_{3}\right\lrcorner X_{5}\right\lrcorner X_{6}\right\lrcorner \tilde{D}_{x}\right\lrcorner \tilde{D}_{t}\right\lrcorner \Omega \\
& =\frac{-4 c_{6}^{3} d u_{x}-4 c_{6}^{2} v v_{x} d v+\left(4 c_{6}^{2} c_{5}-2 c_{6}^{2} v^{2}+v_{x}^{4}\right) d v_{x}}{4 c_{6}^{3}}
\end{aligned}
$$


satisfies

$$
\Omega_{4}=d F_{4}=d\left(-u_{x}+\frac{c_{5} v_{x}}{c_{6}}-\frac{v^{2} v_{x}}{2 c_{6}}+\frac{v_{x}^{5}}{20 c_{6}^{3}}\right) .
$$

Then, on the level manifolds $F_{4}=c_{4}, F_{5}=c_{5}, F_{6}=c_{6}$ we find

$$
\begin{aligned}
\Omega_{3}= & \left.\left.\left.\left.\left.\left.\left.M X_{1}\right\lrcorner X_{2}\right\lrcorner X_{4}\right\lrcorner X_{5}\right\lrcorner X_{6}\right\lrcorner \tilde{D}_{x}\right\lrcorner \tilde{D}_{t}\right\lrcorner \Omega \\
= & \left(40 c_{6}^{4} d u+\left(-20 c_{6}^{3} c_{5}+20 c_{6}^{2} v v_{x}^{2}-5 c_{6} v_{x}^{4}\right) d v+\right. \\
& \left.\left(-40 c_{6}^{3} c_{4}-20 c_{6}^{2} c_{5} v_{x}+20 c_{6}^{2} v^{2} v_{x}-20 c_{6} v v_{x}^{3}+3 v_{x}^{5}\right) d v_{x}\right) /\left(40 c_{6}^{4}\right)
\end{aligned}
$$

and

$\Omega_{3}=d F_{3}=d\left(u+\frac{-40 c_{6}^{3} c_{5} v-80 c_{6}^{3} c_{4} v_{x}-20 c_{6}^{2} c_{5} v_{x}^{2}+20 c_{6}^{2} v^{2} v_{x}^{2}-10 c_{6} v v_{x}^{4}+v_{x}^{6}}{80 c_{6}^{4}}\right)$,

while, on the submanifolds $F_{6}=c_{6}$, we have

$$
\begin{aligned}
\Omega_{2} & \left.\left.\left.\left.\left.\left.\left.=M X_{1}\right\lrcorner X_{3}\right\lrcorner X_{4}\right\lrcorner X_{5}\right\lrcorner X_{6}\right\lrcorner \tilde{D}_{x}\right\lrcorner \tilde{D}_{t}\right\lrcorner \Omega \\
& =\frac{-d v_{x}+c_{6} d x}{c_{6}}
\end{aligned}
$$

so that

$$
\Omega_{2}=d F_{2}=d\left(v_{x}-c_{6} x\right) .
$$

Finally, on the level manifolds $F_{2}=c_{2}, F_{6}=c_{6}$ we have

$$
\begin{aligned}
\Omega_{1} & \left.\left.\left.\left.\left.\left.\left.=M X_{2}\right\lrcorner X_{3}\right\lrcorner X_{4}\right\lrcorner X_{5}\right\lrcorner X_{6}\right\lrcorner \tilde{D}_{x}\right\lrcorner \tilde{D}_{t}\right\lrcorner \Omega \\
& =\frac{-2 c_{6} d t+d v+\left(-c_{6} x-c_{2}\right) d x}{2 c_{6}} \\
& =d F_{1}=d\left(-v-2 c_{6}\left(-\frac{x^{2}}{4}-\frac{c_{2} x}{2 c_{6}}-t\right)\right)
\end{aligned}
$$

and from $F_{1}=c_{1}$ and the previous equations we obtain the explicit solution

$$
\begin{aligned}
v= & -c_{1}-2 c_{6}\left(-\frac{x^{2}}{4}-\frac{c_{2} x}{2 c_{6}}-t\right) \\
u= & \left(-80 c_{6}^{6} t^{2} x^{2}-20 c_{6}^{6} t x^{4}-c_{6}^{6} x^{6}-160 c_{6}^{5} c_{2} t^{2} x-80 c_{6}^{5} c_{2} t x^{3}-6 c_{6}^{5} c_{2} x^{5}+\right. \\
& 80 c_{6}^{5} c_{1} t x^{2}+10 c_{6}^{5} c_{1} x^{4}+80 c_{6}^{4} c_{5} t+40 c_{6}^{4} c_{5} x^{2}+80 c_{6}^{4} c_{4} x-80 c_{6}^{4} c_{2}^{2} t^{2}+ \\
& -80 c_{6}^{4} c_{2}^{2} t x^{2}-10 c_{6}^{4} c_{2}^{2} x^{4}+160 c_{6}^{4} c_{2} c_{1} t x+40 c_{6}^{4} c_{2} c_{1} x^{3}-20 c_{6}^{4} c_{1}^{2} x^{2}+ \\
& -80 c_{6}^{4} c_{3}+80 c_{6}^{3} c_{5} c_{2} x-40 c_{6}^{3} c_{5} c_{1}+80 c_{6}^{3} c_{4} c_{2}+80 c_{6}^{3} c_{2}^{2} c_{1} t+ \\
& 40 c_{6}^{3} c_{2}^{2} c_{1} x^{2}-40 c_{6}^{3} c_{2} c_{1}^{2} x+20 c_{6}^{2} c_{5} c_{2}^{2}+20 c_{6}^{2} c_{2}^{4} t+10 c_{6}^{2} c_{2}^{4} x^{2}+ \\
& \left.-20 c_{6}^{2} c_{2}^{2} c_{1}^{2}+4 c_{6} c_{2}^{5} x-10 c_{6} c_{2}^{4} c_{1}-c_{2}^{6}\right) /\left(80 c_{6}^{4}\right) .
\end{aligned}
$$




\section{References}

[1] M.A. Barco, An application of solvable structures to classical and non classical similarity solutions, J. Math. Phys. 42 (2001) 3714-34

[2] M.A. Barco, Solvable structures and their application to a class of Cauchy problem, Eur. J. Appl. Math. 13 (2002) 449-477

[3] M.A. Barco and G. Prince, Solvable symmetry structures in differential form applications, Acta Appl. Math. 66 (2001) 89-121

[4] M.A. Barco and G. Prince, New symmetry solution techniques for firstorder non-linear PDEs, Appl. Math. Comput. 124 (2001) 169-196

[5] P. Basarab-Horwath, Integrability by quadratures for systems of involutive vector fields, Ukrain. Math. J. 43 (1991) 1236-1242

[6] G.W. Bluman and S. Kumei, Symmetries and Differential Equations (Springer, New York, 1989)

[7] G.W. Bluman and J.D.Cole, The general similarity solution of the heat equation, J. Math. Mech. 18 (1969) 1025-1042

[8] D. Catalano Ferraioli and P. Morando, Local and nonlocal solvable structures in the reduction of ODEs, J. Phys. A: Math Theor 42 (2009) 035210 (15pp)

[9] P. Clarkson and M. Kruskal, New similarity reduction of the Boussinesq equation, J. Math. Phys. 30 (1989) 2201-2213

[10] M.E. Fels, Integrating scalar ordinary differential equations with symmetry revisited, Found. Comput. Math. 417-454 (2007)

[11] G. Gaeta and P. Morando, On the geometry of lambda-symmetries and PDEs reduction, J. Phys. A:Math. Gen. 37 (2004) 6955-6975

[12] V.A. Galaktionov and S.R. Svirshchevskii, Exact solutions and invariant subspaces of nonlinear partial differential equations in mechanicas and physics (Chapman and Hall/CRC, London, 2007)

[13] T. Hartl and C. Athorne, Solvable structures and hidden symmetries, J. Phys. A: Math Gen 27 (1994) 3463-3471

[14] L. Ji, Conditional Lie-Bäcklund symmetries and differential constraints for inhomogeneous nonlinear diffusion equations due to linear determining equations, J. Math. Anal. Appl 440 (2016) 286-299

[15] N. Kamran, R. Milson, and P. J. Olver, Invariant modules and the reduction of nonlinear partial differential equations to dynamical systems, Advances in Mathematics 156 (2000) 286-319 
[16] I.S. Krasil'schik and A.M. Vinogradov eds., Symmetries and conservation laws for differential equations of mathematical physics (A.M.S., Providence, 1999)

[17] B. Kruglikov, Symmetry approaches for reductions of PDEs, differential constraints and Lagrange-Charpit method, Acta Appl. Math. 101 (2008) $145-161$

[18] D.Levi and P. Winternitz, Nonclassical symmetry reduction: example of the Boussinesq equation, J. Phys. A 22 (1989) 2915-2924 (Graduate Texts in Mathematics 220, Springer-Verlag, New York, 2003)

[19] P.J. Olver, Application of Lie groups to differential equations (Springer, Berlin, 1993)

[20] P.J. Olver, Directc reduction and differential constraints, Proc. R. Soc. London A 444 (1994) 509-523

[21] P.J. Olver and P. Rosenau, Group-invariant solutions of differential equations, SIAM J. Appl. Math. 47 (1987) 263-278

[22] E. Pucci and G. Saccomandi, On the reduction methods for ordinary differential equations, J. Phys. A 35 (2002) 6145-6155

[23] E. Pucci and G. Saccomandi, Evolution equations, invariant surface conditions and functional separation of variables, Phisica D 139 (2000) 28-47

[24] J. Sherring and G. Prince, Geometric aspects of reduction of order, Trans. Am. Math. Soc. 334 (1992) 433-453

[25] O. Stormark, Lie's structural approach to PDE systems (Cambridge University Press, Cambridge, 2000)

[26] R.Z. Zhdanov, Conditional Lie-Bäcklund symmetry and reduction of evolution equation, J. Phys. A 28 (1995) 3841-3850 